\title{
Inventário de Emissões de Gases de Efeito Estufa no Campus Gávea da PUC-Rio
}

\author{
Greenhouse Gas Emissions Inventory on Gávea Campus of PUC-Rio
}

João Paulo Andrade F. de Carvalho', Ana Ghislane H. Pereira van Elk², Celso Romanel ${ }^{3}$

\begin{abstract}
RESUMO
O presente trabalho apresenta resultados preliminares de um inventário de emissões de gases de efeito estufa (GEE) elaborado para o campus da PUCRio, situado no bairro da Gávea, zona sul da cidade do Rio de Janeiro. Foram identificadas emissões de GEE nos escopos 1 (combustão estacionária e móvel, emissões fugitivas), escopo 2 (consumo de energia elétrica) e escopo 3 (transporte, viagens, geração e descarte de resíduos sólidos), seguindo metodologias recomendadas pelo Programa Brasileiro GHG Protocol. Dentre as fontes de emissão, a mais difícil de quantificar foi justamente a principal geradora, relacionada com os deslocamentos entre o campus e as residências dos 20 mil potenciais frequentadores diários da PUC-Rio durante 2011.
\end{abstract}

Palavras-chave: inventário de gases de efeito estufa; gerenciamento de emissões; PUC-Rio.

\begin{abstract}
This paper presents the preliminary results of a greenhouse gas (GHG) inventory prepared for the campus of PUC-Rio, located in the district of Gávea, south zone of the city of Rio de Janeiro. GHG emissions have been identified in scope 1 (stationary and mobile combustion, fugitive emissions), scope 2 (electricity consumption) and scope 3 (transport, travel, generation and disposal of solid waste), according to the methodologies recommended by the Brazilian GHG Protocol Program. Among the sources of emissions, the main generator was the most difficult to quantify, and it was related to the commuting between the campus and the residences of estimated 20,000 people who frequented PUC-Rio daily in 2011.
\end{abstract}

Keywords: greenhouse gas inventory; management of emissions; PUC-Rio.

\section{INTRODUÇÃO}

Dentre os problemas ambientais do mundo contemporâneo, as mudanças climáticas são dos mais desafiadores por interferirem na dinâmica dos biomas e afetarem a vida no planeta. O problema necessita de soluções positivas para reduzir o avanço das alterações provocadas pela ação do homem sobre o clima. No entanto, parte das mudanças necessárias passa por alterações de comportamento e hábitos, que podem levar gerações para se concretizarem.

Em dezembro de 2006, foi criada nos Estados Unidos a American College \& University President's Climate Commitment (ACUPCC) com o propósito de reduzir as emissões de gases de efeito estufa (GEE) nos campi universitários, promover esforços de pesquisa e de educação, encorajando jovens a desenvolver soluções sociais, econômicas e tecnológicas que possam reverter o aquecimento global e outros impactos gerados pelas mudanças climáticas. Atualmente, a associação já conta com a participação de mais de 600 instituições.
No Brasil, a PUC-Rio lançou, em 2009, como iniciativa pioneira, a Agenda Ambiental (NIMA, 2015), instrumento que oferece subsídios de gestão para a administração do campus, propondo metas de curto, médio e longo prazos em tópicos como biodiversidade, água, energia, atmosfera, materiais, resíduos e educação ambiental.

O presente trabalho se insere no propósito desta Agenda Ambiental, com a elaboração de um inventário preliminar de emissões de GEE no campus da universidade, no bairro da Gávea, Rio de Janeiro. O inventário foi realizado tomando como base o ano de 2011.

\section{METODOLOGIA}

\section{Coleta de dados}

As diretrizes do Painel Intergovernamental de Mudanças Climáticas (IPCC, 2006) classificam o processo de estimativa de emissões

Mestre em Engenharia Urbana e Ambiental da Pontifícia Universidade Católica do Rio de Janeiro - Rio de Janeiro (RJ), Brasil.

2Doutora em Geotecnia Ambiental pela Universidad de Oviedo, Espanha. Professora Adjunta da Universidade do Estado do Rio de Janeiro (UERJ) - Rio de Janeiro (RJ), Brasil. ${ }^{3}$ Doutor em Engenharia Civil pela University of Arizona. Professor Associado da Pontifícia Universidade Católica do Rio de Janeiro - Rio de Janeiro (RJ), Brasil. Endereço para correspondência: Ana Ghislane H. Pereira van Elk - Rua São Francisco Xavier, 524, Pavilhão João Lyra Filho, 50 andar, sala 5029 , Bloco F - Maracanã - 24550400 - Rio de Janeiro (RJ), Brasil - E-mail: anavanelk@gmail.com

Recebido: 01/02/16 - Aceito: 20/07/16 - Reg. ABES: 155865 
em três níveis distintos, identificados como Tiers. O Tier 1 se refere a uma metodologia básica, o Tier 2 a um processo intermediário, enquanto o Tier 3 é um método mais abrangente, tanto em termos de cálculo quanto na necessidade da quantidade e qualidade dos dados. No presente trabalho, foram aplicados métodos dos Tiers 1 e 2 para elaboração do inventário da PUC-Rio, com a consideração dos seguintes escopos recomendados pela American College \& University President's Climate Commitment (ACUPCC):

\section{Escopo 1 - Emissões diretas de fontes próprias ou controladas pela instituição:}

\section{Combustão estacionária e móvel}

O controle da maioria dos veículos próprios da universidade é de responsabilidade da prefeitura do campus, com estimativa do consumo de combustível anual em 10.000 litros. A frota de ônibus destinada para o transporte de funcionários é gerenciada pela Associação de Funcionários da PUC-Rio. Considerando que os itinerários dos ônibus são fixos, foi utilizada a ferramenta Google Maps para determinação dos quilômetros percorridos em cada trajeto. Em 2011, o número de dias de trabalho dos funcionários foi 215 , com base no calendário administrativo da universidade.

\section{Emissões fugitivas}

Foram analisados os equipamentos, com seus respectivos gases de efeito estufa, por meio do método utilizado nos processos de recarga e manutenção dos aparelhos instalados no campus (ar-condicionado, bebedouros, geladeiras, extintores de incêndio).

De acordo com informações da prefeitura do campus, o total de equipamentos de ar-condicionado instalados foi de 1.328, sendo 829 aparelhos de janela, 10 geradores de ar-condicionado central e 489 aparelhos de ar-condicionado tipo split. Além desses equipamentos, havia ainda 80 bebedouros e aproximadamente 100 geladeiras. O gás predominante em aparelhos de ar-condicionado, bebedouros e geladeiras é o HCFC-22 (clorodifluorometano), um gás controlado pelo Protocolo de Montreal, assinado pelo Brasil e, portanto, não incluído no Protocolo de Quioto.

O campus da PUC-Rio contava, em 2011, com 52 extintores de incêndio de $4 \mathrm{~kg}$ e 296 extintores de $6 \mathrm{~kg}$. Na manutenção periódica anual, os extintores são descarregados e $90 \%$ dos gases são transferidos para outros cilindros que retornam aos distribuidores. Os $10 \%$ restantes são liberados para a atmosfera. Assim, cada extintor de $4 \mathrm{~kg}$ de capacidade libera $0,4 \mathrm{~kg}$ para a atmosfera e cada extintor de $6 \mathrm{~kg}$ libera $0,6 \mathrm{~kg}$. O total de emissões considerando os 348 extintores existentes foi estimado em $198,4 \mathrm{~kg}$ de $\mathrm{CO}_{2}$.

\section{Escopo 2 - Emissões de GEE na geração da eletricidade adquirida e consumida pela instituição}

Energia elétrica

O consumo de energia elétrica na PUC-Rio foi levantado junto à prefeitura do campus, constatando-se um consumo mensal médio de 34.000 kWh (CARVALHO, 2013). Para o inventário, adotou-se a média dos fatores de emissão mensais na geração de eletricidade divulgados pelo SIN - Sistema Interligado Nacional (BRASIL, 2013). Os fatores de emissão médios na geração de energia elétrica estimam a quantidade de $\mathrm{CO}_{2}$ considerando o conjunto de todas as usinas hidrelétricas existentes no país. A adoção desses fatores de emissão implica que a energia elétrica consumida foi basicamente hidrelétrica.

\section{Escopo 3 - Emissões indiretas cujas fontes não pertencem à universidade ou não são por ela controladas}

\section{Transporte}

As estimativas de emissões GEE em transporte rodoviário podem ser baseadas em duas informações independentes: quantidade de combustível consumido ou distância percorrida pelo veículo. Neste trabalho, foi considerada a quantidade de combustível consumida utilizando a Equação 1 :

$E=\sum \operatorname{com} b_{a b c} \times F E_{a b c}$

Em que:

$E$ representa a quantidade $(\mathrm{kg})$ de emissões de $\mathrm{CH}_{4}$ e $\mathrm{N}_{2} \mathrm{O}$, comb a quantidade de combustível consumido (TJ),

$F E$ um fator de emissão (kg/TJ) e os subscritos $a, b, c$ estão associados ao tipo de combustível, tipo de veículo e tecnologia do veículo, respectivamente.

Os fatores de emissão para cálculo das emissões de $\mathrm{CH}_{4}$ e $\mathrm{N}_{2} \mathrm{O}$ foram apresentados nas diretrizes para Inventários Nacionais de Gases de Efeito Estufa (IPCC, 2006), bem como fatores de emissão por frota e tipo de combustível no Segundo Inventário Brasileiro de Emissões Antrópicas de Gases de Efeito Estufa (BRASIL, 2010).

Inicialmente buscaram-se dados na universidade sobre o número de potenciais frequentadores (Tabela 1) e a frequência diária nos estacionamentos externo (alunos e visitantes, com uso médio diário de 2.300 carros e 110 motos) e interno (professores e funcionários com uso médio diário de 545 carros).

Também foi realizada uma pesquisa com frequentadores do campus para obtenção de informações específicas, necessárias para a quantificação das emissões de transporte, como distâncias percorridas entre locais de residência e a universidade, tipo de combustível utilizado no veículo, ano de fabricação do veículo, número de dias da semana que frequenta o 
campus etc. Nessa etapa, os questionários foram respondidos por 479 pessoas, em sua maioria por alunos em diferentes horários de funcionamento da universidade, entre $7 \mathrm{~h}$ e $22 \mathrm{~h}$. A margem de erro da pesquisa foi de aproximadamente $4,48 \%$, considerando um intervalo de confiança de $95 \%$.

As informações da pesquisa indicaram que 71,2 \% dos alunos e visitantes utilizaram o estacionamento externo, enquanto $28,8 \%$ deles estacionaram o veículo em outros locais, geralmente ruas vizinhas. Assim, o número total diário de veículos de alunos e visitantes foi de 2.963 unidades, correspondente à soma dos veículos controlados pelo estacionamento externo (2.300 unidades), mais um adicional estimado em 28,8\% (663 unidades).

O estacionamento interno foi ocupado exclusivamente por professores e funcionários, correspondendo a 545 veículos diários.

Outro dado relevante refere-se aos deslocamentos efetuados por meio de táxi. De acordo com a Cooperativa de Táxi Unigávea, que opera junto ao portão principal da universidade, são feitas aproximadamente 550 viagens diárias originadas da PUC-Rio.

Para a entrada de dados na planilha de cálculo foi ainda necessário saber a distância anual percorrida pelos veículos, o ano de fabricação dos mesmos e o tipo de combustível utilizado. A distância média percorrida pelos frequentadores da universidade nos deslocamentos diários entre a

Tabela 1 - Número de potenciais frequentadores diários do campus da PUC-Rio.

\begin{tabular}{|c|c|c|}
\hline Frequentadores do campus em 2011 & $\mathrm{~N}^{\circ}$ & Fonte \\
\hline Alunos de graduação & 13.293 & $\begin{array}{l}\text { Vice-Reitoria } \\
\text { Acadêmica }\end{array}$ \\
\hline Alunos de pós-graduação & 2.291 & $\begin{array}{l}\text { Vice-Reitoria } \\
\text { Acadêmica }\end{array}$ \\
\hline Funcionários & 1.591 & $\begin{array}{l}\text { Vice-Reitoria } \\
\text { Administrativa }\end{array}$ \\
\hline Professores & 1.324 & $\begin{array}{l}\text { Vice-Reitoria } \\
\text { Administrativa }\end{array}$ \\
\hline $\begin{array}{l}\text { Terceirizados (limpeza, } \\
\text { manutenção, estacionamento etc.) }\end{array}$ & 350 & Estimativa \\
\hline $\begin{array}{l}\text { Funcionários de serviços } \\
\text { (lojas, restaurantes, bancos etc.) }\end{array}$ & 150 & Estimativa \\
\hline $\begin{array}{l}\text { Visitantes (serviços de } \\
\text { entrega, eventos, convidados etc.) }\end{array}$ & 700 & Estimativa \\
\hline Total & 19.699 & \\
\hline
\end{tabular}

residência e o campus foi determinada considerando uma média ponderada das respostas obtidas pela pesquisa executada, resultando em uma distância média de $34 \mathrm{~km}$ para trajetos de ida e volta. O número total de quilômetros percorridos no ano foi obtido multiplicando-se essa distância (34 km) pelo número de dias que os usuários de veículos frequentaram o campus. Como a presença média dos alunos no campus foi de 4,7 dias por semana, conforme levantamento da pesquisa, o número anual de dias foi proporcionalmente reduzido de 175 , do calendário acadêmico, para 165 dias.

A distribuição dos anos de fabricação dos veículos foi considerada igualmente para os automóveis de alunos, professores, táxis e motocicletas. O tipo de combustível (5\% álcool, $87 \%$ gasolina, $2 \%$ diesel e $6 \%$ GNV) foi aplicado aos automóveis de professores e funcionários (Tabela 2) e automóveis de alunos e visitantes (Tabela 3), enquanto os táxis (Tabela 4) foram admitidos com $100 \%$ de abastecimento por gás natural veicular (GNV) e as motocicletas (Tabela 5) com 100\% de abastecimento por gasolina. Os deslocamentos em táxi e motocicletas foram considerados efetuados apenas por alunos.

Outro dado importante levantado na pesquisa é que aproximadamente $60 \%$ dos frequentadores chegam à universidade utilizando ônibus públicos; $24,5 \%$ são transportados por automóveis ou motos e os $15,5 \%$ restantes se locomoveram a pé ou de bicicleta. Parte dos funcionários da instituição chega e retorna da universidade por meio de ônibus fretados, cujas emissões já foram consideradas no escopo 1.

Seguindo o mesmo procedimento feito para o cálculo das distâncias percorridas pelos usuários de automóveis, foi possível obter a soma das distâncias percorridas anualmente por ônibus públicos, multiplicando o número de 11.819 pessoas (60\% dos 19.699 potenciais frequentadores) pelo número de dias presentes no campus (165, admitindo que a maioria dessas é formada por estudantes) e pela distância média percorrida de $34 \mathrm{~km}$. Considerando que um ônibus transporta em média 70 passageiros, a parcela referente à contribuição dos usuários de ônibus foi equivalente a $947.208 \mathrm{~km}(11.819$ x 165 x 34 / 70). A idade média adotada para a frota de ônibus foi 6 anos, conforme dado divulgado pela Associação Nacional das Empresas de Transportes Urbanos (NTU, 2015).

\section{Viagens a trabalho}

Este item refere-se a viagens realizadas por professores, alunos ou funcionários da PUC-Rio, motivados pela atividade acadêmica. No caso

Tabela 2 - Deslocamentos em automóveis - professores e funcionários.

\begin{tabular}{l|c|c|c|c|c|c|c|c} 
Ano de fabricação & $\%$ & $\begin{array}{c}\text { Total de } \\
\text { veículos }\end{array}$ & Gasolina & $\begin{array}{c}\text { Distância média } \\
\text { percorrida no ano } \\
(\mathrm{km}) \text { - gasolina }\end{array}$ & $\begin{array}{c}\text { Aistância média } \\
\text { percorrida no ano } \\
(\mathrm{km}) \text { - álcool }\end{array}$ & $\begin{array}{c}\text { Distância média } \\
\text { GNV / diesel } \\
\text { percorrida no ano } \\
(\mathrm{km})-\text { GNV / Diesel }\end{array}$ \\
\hline 2011 & 44 & 240 & 209 & 1.527 .790 & 12 & 87.720 & 19 & 138.890 \\
\hline $2010-2009$ & 30 & 163 & 142 & 1.038 .020 & 8 & 58.480 & 13 & 95.030 \\
\hline $2008-2007$ & 8 & 44 & 38 & 321.640 & 2 & 14.620 & 4 & 29.240 \\
\hline $2006-2005$ & 9 & 49 & 43 & 314.330 & 3 & 21.930 & 3 & 21.930 \\
\hline$<2005$ & 9 & 49 & 43 & 314.330 & 3 & 21.930 & 3 & 21.930 \\
\hline Total & 100 & 545 & 475 & & 28 & & 42 & \\
\hline
\end{tabular}


de viagens aéreas a metodologia utilizada pelo Programa Brasileiro GHG Protocol utiliza uma abordagem Tier 1 e adota fatores de emissão(FE) médios para $\mathrm{CO}_{2}, \mathrm{CH}_{4}$ e $\mathrm{N}_{2} \mathrm{O}$, apresentados pelo Department for Environment, Food and Rural Affairs (DEFRA, 2013) do Reino Unido. O cálculo das emissões de $\mathrm{CO}_{2}, \mathrm{CH}_{4}$ e $\mathrm{N}_{2} \mathrm{O}$ é feito de maneira direta aplicando a Equação 2:

$E=D \times F_{c} \times F E$

Em que:

$E$ representa a quantidade $(\mathrm{kg})$ de emissões de cada gás,

$D$ a distância aérea percorrida,

$F_{c}$ o fator de correção de rota, com valor padrão de 1,09, e

FE o fator de emissão correspondente a cada gás.

Para obter as informações relacionadas com viagens foram solicitados dados à agência de viagens contratada pela universidade e responsável pela emissão dos bilhetes aéreos. Em 2011, foram emitidos 615 bilhetes; para cada trajeto foram determinadas as distâncias aéreas entre as cidades, estimadas com auxílio do aplicativo Google Earth.

\section{Resíduos sólidos}

Os aterros sanitários produzem quantidades significativas de $\mathrm{CH}_{4}, \mathrm{CO}_{2} \mathrm{e}$ pequenas quantidades de $\mathrm{N}_{2} \mathrm{O}$. Um método Tier 2, geralmente utilizado para a estimativa das emissões provenientes de aterros, é o modelo de decaimento de primeira ordem (IPCC, 2006), que considera uma emissão persistente de $\mathrm{CH}_{4}$ ao longo de vários anos após o descarte de resíduos no local de disposição final. Para esta categoria, obteve-se informações de um estudo que teve o objetivo de elaborar um plano de gerenciamento dos resíduos sólidos do campus da PUC (GOMES, 2012).

Em 2011, a PUC-Rio enviou para a Central de Tratamento de Resíduos Sólidos (CTR) de Nova Iguaçu 1.591 toneladas de resíduos sólidos, cuja composição consistia de $26 \%$ de papel e papelão, $6 \%$ de plástico rígido, 3\% de plástico fino, $1 \%$ de alumínio, $26 \%$ de matéria orgânica, 31\% de resíduos de jardim e 7\% de materiais inertes. As emissões provenientes dos resíduos sólidos foram contabilizadas em função da distância anual percorrida pelos veículos para conduzir os resíduos do campus até o aterro sanitário, totalizando $12.700 \mathrm{~km}$.

\section{RESULTADOS}

Os dados anteriormente coletados para a estimativa das emissões de GEE foram aplicados à ferramenta de cálculo do programa Brasileiro GHG Protocol (FGV, 2015), convertendo cada gás de efeito estufa em dióxido de carbono equivalente $\left(\mathrm{CO}_{2 \mathrm{e}}\right)$, em função do seu potencial de aquecimento global (PAG). Esses dados estão apresentados na Tabela 6, considerando o PAG de 21 para $\mathrm{CH}_{4}, 310$ para $\mathrm{N}_{2} \mathrm{O}$ e 1.300 para HFCs.

Emissões resultantes da combustão de biomassa devem ser tratadas de forma diferente daquelas provenientes de combustíveis fósseis, uma vez que a quantidade de dióxido de carbono liberada na combustão é igual àquela retirada da atmosfera durante o processo de fotossíntese e pode ser considerada neutra. Valores significativos de emissões de $\mathrm{CO}_{2}$ advindas da combustão da biomassa estão reportados separadamente (Tabela 7)

Tabela 3 - Deslocamentos em automóveis - alunos e visitantes.

\begin{tabular}{l|c|c|c|c|c|c|c|c} 
Ano de fabricação & $\%$ & $\begin{array}{c}\text { Total de } \\
\text { veículos }\end{array}$ & Gasolina & $\begin{array}{c}\text { Distância média } \\
\text { percorrida no ano } \\
(\mathrm{km})-\text { gasolina }\end{array}$ & $\begin{array}{c}\text { Distância média } \\
\text { percorrida no ano } \\
(\mathrm{km}) \text { - álcool }\end{array}$ & $\begin{array}{c}\text { Distância média } \\
\text { GNV/Diesel }\end{array}$ \\
$\begin{array}{l}\text { percorrida no ano } \\
(\mathrm{km})-\text { GNV/Diesel }\end{array}$ \\
\hline 2011 & 44 & 1303 & 1134 & 6.361 .740 & 65 & 364.650 & 104 & 583.440 \\
\hline $2010-2009$ & 30 & 889 & 773 & 4.336 .530 & 45 & 252.450 & 71 & 398.310 \\
\hline $2006-2005$ & 8 & 237 & 206 & 1.155 .660 & 12 & 67.320 & 19 & 106.590 \\
\hline$<2005$ & 9 & 267 & 232 & 1.301 .520 & 13 & 72.930 & 22 & 123.420 \\
\hline Total & 100 & 2963 & 2577 & 1.301 .520 & 13 & 72.930 & 22 & 123.420 \\
\hline
\end{tabular}

Tabela 4 - Deslocamentos em táxis.

\begin{tabular}{l|c|c|c|c}
$\begin{array}{l}\text { Ano de } \\
\text { fabricação }\end{array}$ & $\%$ & $\begin{array}{c}\text { Total de } \\
\text { veículos }\end{array}$ & GNV & $\begin{array}{c}\text { Distância média percorrida } \\
\text { no ano }(\mathrm{km})-\text { GNV }\end{array}$ \\
\hline 2011 & 44 & 242 & 242 & 1.357 .620 \\
\hline $2010-2009$ & 30 & 165 & 165 & 925.650 \\
\hline $2008-2007$ & 8 & 43 & 43 & 241.230 \\
\hline $2006-2005$ & 9 & 50 & 50 & 280.500 \\
\hline$<2005$ & 9 & 50 & 50 & 280.500 \\
\hline Total & 100 & 550 & 550 & \\
\hline
\end{tabular}

Tabela 5 - Deslocamentos em motocicletas.

\begin{tabular}{l|c|c|c}
$\begin{array}{l}\text { Ano de } \\
\text { fabricação }\end{array}$ & $\%$ & $\begin{array}{c}\text { Total de veículos } \\
\text { (gasolina) }\end{array}$ & $\begin{array}{c}\text { Distância média percorrida } \\
\text { no ano }(\mathrm{km})\end{array}$ \\
\hline 2011 & 44 & 48 & 269.280 \\
\hline $2010-2009$ & 30 & 33 & 185.130 \\
\hline $2008-2007$ & 8 & 9 & 50.490 \\
\hline $2006-2005$ & 9 & 10 & 56.100 \\
\hline$<2005$ & 9 & 10 & 56.100 \\
\hline Total & 100 & 110 & \\
\hline
\end{tabular}


Tabela 6 - Emissões de GEE nos escopos 1, 2 e 3.

\begin{tabular}{l|c|c|c|c|c|c}
\multirow{2}{*}{$\mathrm{GEE}$} & \multicolumn{3}{|c|}{ Emissões em } & \multicolumn{3}{c}{ Emissões em toneladas métri- } \\
cas de $\mathrm{CO}_{2}$ equivalente $\left(\mathrm{tCO}_{2} \mathrm{e}\right)$
\end{tabular}

Tabela 7 - Emissões de GEE por combustão de biomassa.

\begin{tabular}{c|c|c|c|c|c|c|}
\multirow{2}{*}{ GEE } & \multicolumn{3}{|c|}{$\begin{array}{c}\text { Emissões em } \\
\text { toneladas métricas }\end{array}$} & \multicolumn{2}{c|}{ Emissões em toneladas métri- } \\
cas de $\mathrm{CO}_{2}$ equivalente $\left(\mathrm{tCO}_{2} \mathrm{e}\right)$
\end{tabular}

e devem-se, em grande parte, à composição dos combustíveis, com teores de etanol e biodiesel. A recuperação do metano $\left(\mathrm{CH}_{4}\right)$ no aterro sanitário CTR de Nova Iguaçu também contribuiu para a obtenção desses resultados.

\section{CONCLUSÕES}

O inventário é o passo inicial para a elaboração de um programa de gerenciamento das emissões de GEE. Uma vez determinadas as fontes e seus potenciais de emissão é possível planejar métodos e ações para alcançar a redução desejada. No caso especial de universidades, essa iniciativa é também relevante como parte da formação educacional dos alunos, incentivando-os para o desenvolvimento de novas soluções que possam prevenir e combater o aquecimento global, bem como outros impactos decorrentes das mudanças climáticas.

Os valores obtidos nesse inventário mostraram que as emissões de escopo 3, na categoria transporte, representaram mais de $98 \%$ das emissões totais no campus da PUC-Rio, devido aos deslocamentos diários entre a universidade e os locais de residência dos 20 mil frequentadores do campus. As emissões do escopo 1 foram pouco significativas, em grande parte, devido à pequena frota de veículos próprios da universidade, enquanto as emissões do escopo 2 também foram reduzidas em razão das características da matriz energética brasileira, predominantemente de origem hídrica. A arquitetura dos edifícios da universidade, assim como as características do espaço do campus da Gávea, muito arborizado e ventilado, também contribuiu para uma menor utilização de aparelhos de ar-condicionado e iluminação artificial.

O presente inventário de GEE indica que um projeto para estimular caronas solidárias, possibilidade de isenção do pagamento de estacionamento para automóveis com mais de três passageiros transportados e incentivos para a utilização de bicicletas pelos alunos, funcionários e professores, são exemplos de possíveis soluções de transporte, para atingir metas de redução de emissão de GEE no campus.

\section{REFERÊNCIAS}

BRASIL. Ministério da Ciência e Tecnologia. (2010) Segundo Inventário Brasileiro de Emissões Antrópicas de Gases de Efeito Estufa. Relatórios de Referência. Brasília, DF. Disponível em: <www. mct.gov.br/upd_blob/O212/212594.pdf>. Acesso em: 25 jun. 2015.

BRASIL. Ministério da Ciência e Tecnologia. (2013) Sistema Interligado Nacional (SIN). Disponível em: <www.mct.gov.br/index. php/content/view/321144.html>. Acesso em: 12 set. 2015.

CARVALHO, J.P.A.F. (2013) Gerenciamento de emissões de gases de efeito estufa do campus Gávea da PUC-Rio: inventário e proposta de mitigação de emissões. Dissertação (Programa de Mestrado Profissional em Engenharia Urbana e Ambiental) - PUC-Rio, Rio de Janeiro. 110p.

DEFRA - Department for Environment, Food and Rural Affairs (UK). (2013) Government GHG Conversion Factors for Company Reporting: Methodology Paper for Emission Factors. Disponível em: <www.gov.uk/government/uploads/system/uploads/attachment_ data/file/224437/pb13988-emission-factor-methodology-130719. pdf>. Acesso em: 03 jul. 2015.
FGV - Fundação Getúlio Vargas. (2015) Programa Brasileiro GHG Protocol. Disponível em: <www.ghgprotocolbrasil.com.br>. Acesso em: O3 ago. 2015.

GOMES, P.C.G. (2012) Plano de gestão de resíduos sólidos do campus Gávea da PUC-Rio: elaboração, implementação e diagnóstico de operação. Dissertação (Programa de Mestrado Profissional em Engenharia Urbana e Ambiental) - PUC-Rio, Rio de Janeiro. 394p.

IPCC - Intergovernmental Panel on Climate Change. (2006) 2006 IPCC Guidelines for National Greenhouse Gas Inventories. IGES, Japan.

NIMA - Núcleo Interdisciplinar de Meio Ambiente da PUC-Rio. (2015) Agenda Ambiental. Disponível em: <www.nima.puc-rio.br/ noticias/agenda_ambiental.pdf>. Acesso em: 19 dez. 2015.

NTU - Assoc. Nacional de Empresas de Transporte Urbano. (2015) Dados do Transporte Público. Disponível em: <www.ntu.org.br/ novo/ListaDadosPorRegiao.aspx?idArea=7\&idSegundoNivel=17>. Acesso em: 19 dez. 2015 